Pensamiento Crítico Vol. 20 N 1, pp. 125-144

\title{
Plan de utilidades y cash flow
}

\author{
Profits plan and cash flow \\ [Recepción: Marzo de 2015/ Conformidad: Mayo 2015] \\ Raimundo Renaun Pacheco Mexzon*
}

\section{RESUMEN}

Cuando una empresa produce para el mercado bienes o servicios debe invertir fondos en capital de trabajo a la espera de obtener ganancias. Para estimar si esta actividad le genera utilidades es necesario elaborar el presupuesto operativo como un plan de utilidades. Como el Gerente financiero es el responsable de administrar eficientemente los fondos generados por las operaciones de la empresa surge la inquietud de preparar el Cash Flow (Flujo de Caja) a partir del Plan de utilidades. Por lo tanto es objetivo del presente artículo detallar como preparar el Flujo de Caja o Cash Flow a partir del Plan de Utilidades.

Palabras clave: Plan de Utilidades, presupuesto operativo, cash flow.

Clasificación JEL: G10

* Ingeniero economista por la Universidad Nacional de Ingeniería. Magíster en Economía con mención en Finanzas y Doctor en Economía por la Universidad Nacional Mayor de San Marcos 


\title{
Pensamiento Crítico Vol. 20. NoI
}

\begin{abstract}
When a company produces goods or services must invest funds in working capital waiting for profit. To estimate whether this activity generates profits is necessary to develop the operating budget as a Profits Plan. As Finance Manager responsible for managing efficiently the funds generated by the operations of the company, musts prepare the Cash Flow from Profit Plan. Therefore it is objective of this article submit how prepare the Cash Flow from a Profits Plan.
\end{abstract}

Keywords: Profits plan, operating budget, cash flow.

\section{Plan de utilidades}

Una empresa para producir bienes o servicios que saca al mercado combina los factores productivos mano de obra, materiales o insumos y capital reflejado en maquinaria y equipo.

En el proceso productivo la mano de obra puede ser clasificada como mano de obra directa y mano de obra indirecta. Los materiales o insumos también siguen el mismo principio, es decir pueden ser materiales directos o materiales indirectos. Los otros costos y gasto de fabricación que no son mano de obra ni materiales se les consideran como otros costos o gastos indirectos de fabricación.

Por lo tanto el costo de fabricación o producción de bienes o servicios que produce una empresa queda resumido en: mano de obra directa (MOD) más materiales directos (MATD) más costos indirectos de fabricación (CIF).

Por ejemplo si la mano de obra directa para producir los bienes o servicios es $\$$ 200,000 . Los materiales directos llegan a $\$ 500,000$. Y los costos y gastos indirectos de fabricación llegan a $\$ 300,000$ dentro de los cuales la mano de obra indirecta es $\$ 50,000$, los materiales indirectos $\$ 90,000$ y los otros costos indirectos $\$ 160,000$, el costo de fabricación o producción se presentaría en resumen de la siguiente manera: 


\section{Raimundo Pacheco Mexzon}

\section{COSTO DE FABRICACIÓN O PRODUCCIÓN}

\begin{tabular}{|l|c|}
\hline \multicolumn{1}{|c|}{ RUBROS } & MONTO EN \$ \\
\hline MOD & 200,000 \\
\hline MATD & \multicolumn{2}{c|}{500,000} \\
\hline CIF & 500,000 \\
\hline Mano de obra indirecta (MOind) & 90,000 \\
\hline Materiales indirectos (MATind) & 160,00 \\
\hline Otros costos indirectos (OCind) \\
\hline $\begin{array}{c}\text { TOTAL COSTO DE FABRICACIÓ O COSTO } \\
\text { DE PRODUCCIÓN }\end{array}$ & $1^{\prime} 000,000$ \\
\hline
\end{tabular}

Como se puede apreciar en este caso el costo de fabricación o costo de producción llega a $\$ 1 ’ 000,000$.

Para recuperar esta inversión que se realiza para producir un bien o un servicio, la empresa debe vender en el mercado éste bien o servicio producido. Si lo vende y obtiene \$1'500,000 entonces se está ganando \$500,000 como utilidad bruta.

Si a esta utilidad bruta le quitamos los gastos operativos, los gastos financieros y el impuesto a la renta, llegamos a la utilidad neta.

Para determinar si podemos llegar a esta utilidad neta en el próximo periodo debemos preparar el presupuesto operativo como un plan de utilidades.

El Plan de Utilidades o presupuesto operativo de la empresa se elabora tomando en cuenta las políticas, objetivos y metas generalmente para el próximo año.

El Plan de Utilidades está formado por los siguientes presupuestos:

- Presupuesto de ingresos.

- Presupuesto de producción.

- Presupuesto de gastos generales. 


\section{Pensamiento Crítico Vol. 20. NoI}

El presupuesto de ingresos, llamado también presupuesto de ventas se elabora tomando en cuenta las políticas de ventas y las políticas de precios.

Las políticas de ventas definen si se vende toda la producción al contado, al crédito, o una parte al contado y la otra parte al crédito. Y las políticas de precios si éstos son iguales o diferentes para las ventas al contado y para las ventas al crédito.

Como el ingreso es el producto del precio unitario multiplicado por la cantidad se pueden calcular los ingresos diariamente, semanalmente, mensualmente, trimestralmente, semestralmente o anualmente.

Si la programación es trimestral podría utilizarse el siguiente formato:

\section{PRESUPUESTO DE VENTAS}

\begin{tabular}{|c|c|c|c|c|c|c|c|c|c|c|c|c|c|c|c|c|}
\hline \multirow[t]{2}{*}{ RUBROS } & \multirow[t]{2}{*}{ UND } & \multicolumn{3}{|c|}{ 1ER TRM } & \multicolumn{3}{|c|}{ 2DO TRM } & \multicolumn{3}{|c|}{ 3ER TRM } & \multicolumn{3}{|c|}{ 4TO TRM } & \multicolumn{3}{|c|}{ TOTAL AÑO } \\
\hline & & $\mathrm{Pu}$ & $\mathbf{Q}$ & $Y$ & $\mathrm{Pu}$ & $\mathbf{Q}$ & $Y$ & $\mathrm{Pu}$ & $\mathbf{Q}$ & $Y$ & $\mathrm{Pu}$ & $\mathbf{Q}$ & $Y$ & $\mathrm{Pu}$ & $\mathbf{Q}$ & YT \\
\hline Venta & TM & 5 & 10 & 50 & 5 & 10 & 50 & 6 & 10 & 60 & 6 & 10 & 60 & 5.5 & 40 & 220 \\
\hline Ventas al crédito & TM & 6 & 5 & 30 & 6 & 5 & 30 & 7 & 5 & 35 & 7 & 5 & 35 & 6.5 & 20 & 130 \\
\hline TOTAL & & 5.3 & 15 & 80 & 5.3 & 15 & 80 & 6.3 & 15 & 95 & 6.3 & 15 & 95 & 5.8 & 60 & 350 \\
\hline
\end{tabular}

\section{Donde:}

UND = Unidad

$\mathrm{TM}=$ Tonelada métrica

1ER TRM = Primer trimestre

2DO TRM = Segundo trimestre

3ER TRM $=$ Tercer trimestre

4TO TRM = Cuarto trimestre

$\mathrm{Pu}=$ Precio unitario

$\mathrm{Q}=$ Cantidad

$\mathrm{Y}=$ Ingreso trimestral

$\mathrm{YT}=$ Ingreso total del año 


\section{Raimundo Pacheco Mexzon}

Se puede observar en el presupuesto de ventas que la empresa tiene como políticas vender al contado y al crédito con precios diferenciados. Para las ventas al crédito los precios unitarios son mayores.

También es importante mencionar que las ventas al contado trimestralmente llegan a 10 TM y al año a 40 TM. Mientras que las ventas al crédito llegan a 5 TM trimestralmente y al año a $20 \mathrm{TM}$. Es decir las ventas al crédito corresponden a la mitad de las ventas al contado.

Si comparamos los ingresos anuales por ventas que llegan en total a 350, el precio unitario ponderado que esta presupuestando la empresa llega a 5.83 que resulta de dividir este ingreso entre las $60 \mathrm{TM}$ que espera vender en el año $(350 / 60=5.83)$.

El presupuesto de producción se elabora tomando en cuenta las ventas programadas en el presupuesto de ingresos, las políticas de inventarios y los coeficientes técnicos de producción.

Las ventas programadas en el presupuesto de ingresos o de ventas determinan para el presupuesto de producción los requerimientos de la gerencia de comercialización para las ventas.

Las políticas de inventarios determinan cual debe ser el inventario inicial y final para cada año y para cada periodo en que se divide el presupuesto anual de producción.

Y los coeficientes técnicos de producción determinan la relación que existe entre los factores de la producción como son la mano de obra directa, los materiales directos y los gastos indirectos de fabricación con las unidades a producir.

Al igual que para el presupuesto de ventas se está considerando el año en periodos trimestrales.

El formato que se puede utilizar para este presupuesto de producción sería el siguiente: 
Pensamiento Crítico Vol. 20. Nº I

\section{PRESUPUESTO DE PRODUCCIÓN}

\begin{tabular}{|c|c|c|c|c|c|c|c|c|c|c|c|c|c|c|c|c|}
\hline \multirow[t]{2}{*}{ RUBROS } & \multirow[t]{2}{*}{ UND } & \multicolumn{3}{|c|}{ 1ER TRM } & \multicolumn{3}{|c|}{ 2DO TRM } & \multicolumn{3}{|c|}{ 3ER TRM } & \multicolumn{3}{|c|}{ 4TO TRM } & \multicolumn{3}{|c|}{ TOTAL AÑO } \\
\hline & & $\mathrm{Cu}$ & $\mathrm{Q}$ & C & $\mathrm{Cu}$ & $\mathrm{Q}$ & C & $\mathrm{Cu}$ & Q & C & $\mathrm{Cu}$ & $\mathrm{Q}$ & C & $\mathrm{Cu}$ & $\mathrm{Q}$ & CT \\
\hline MOD & $\mathrm{HH}$ & 1 & 11 & 11 & 1 & 15 & 15 & 1.5 & 15 & 22.5 & 1.5 & 19 & 28.5 & 1.28 & 60 & 77 \\
\hline MATD & $\mathrm{TM}$ & 1.5 & 22 & 33 & 1.5 & 30 & 45 & 2 & 30 & 60 & 2 & 38 & 76 & 1.78 & 120 & 214 \\
\hline GIF & $\$$ & & & 5 & & & 5 & & & 5 & & & 5 & & & 20 \\
\hline Cost. Pro. & & 4.45 & & 49 & 4.33 & & 65 & 5.83 & 87 & 7.5 & 5.76 & 10 & 09.5 & 5.18 & 60 & 311 \\
\hline Inv. Inic. & $\mathrm{TM}$ & & 10 & & & 6 & & & 6 & & & 6 & & & 10 & \\
\hline Req. Ventas & $\mathrm{TM}$ & & 15 & & & 15 & & & 15 & & & 15 & & & 60 & \\
\hline Inv. Fin. & $\mathrm{TM}$ & & 6 & & & 6 & & & 6 & & & 10 & & & 10 & \\
\hline Unds a prod & TM & & 11 & & & 15 & & & 15 & & & 19 & & & 60 & \\
\hline
\end{tabular}

\section{Donde:}

MOD = Mano de abra directa

MATD $=$ Materiales directos

GIF $=$ Gastos indirectos de producción o fabricación

Cost. Pro. $=$ Costo de producción

$\mathrm{UND}=$ Unidad

$\mathrm{HH}=$ Hora hombre

$\mathrm{TM}=$ Tonelada métrica

$\$=$ Signo monetario

1ER TRM $=$ Primer trimestre

2DO TRM = Segundo trimestre

3ER TRM $=$ Tercer trimestre

4TO TRM $=$ Cuarto trimestre

$\mathrm{Cu}=$ Costo unitario

$\mathrm{Q}=$ Cantidad

$\mathrm{C}=$ Costo trimestral

$\mathrm{CT}=$ Costo total del año 


\section{Raimundo Pacheco Mexzon}

Inv. Inic. = Inventario inicial

Req. Ventas $=$ Requerimientos de comercialización para ventas

Inv. Fin. = Inventario final

Unds a prod $=$ Unidades a producir

Para elaborar el presupuesto de producción se parte de las unidades a producir en cada trimestre. Se toma en cuenta la política de inventarios y los requerimientos para ventas.

En el caso que se muestra como presupuesto de producción en el cuadro anterior, la política de inventarios se ha definido de la siguiente manera:

- El inventario inicial de productos terminados para cada año será de $10 \mathrm{TM}$.

- El inventario final para cada trimestre será el $40 \%$ de los requerimientos de ventas del próximo trimestre.

La primera política "El inventario inicial de productos terminados para cada año será 10 TM" nos indica que el inventario final para cada año también será de 10 TM, porque se toma en cuenta que el inventario inicial de un año es igual al inventario final del año anterior.

La segunda política "El inventario final para cada trimestre será el $40 \%$ de los requerimientos de ventas del próximo trimestre" nos indica que el inventario final del primer trimestre será de $6 \mathrm{TM}$, porque el $40 \%$ de los requerimientos de ventas del segundo trimestre que es de $15 \mathrm{Tm}$, se calcula multiplicando $0.40 \times 15=6$.

Por lo tanto como el inventario inicial es $10 \mathrm{TM}$, los requerimientos de ventas 15 TM y el inventario final $6 \mathrm{TM}$ para el primer trimestre, las unidades a producir se calculan sumando los requerimientos de ventas con el inventario final y restando a esta suma el inventario inicial lo que nos da $11 \mathrm{TM}$ a producir en el primer trimestre $(15+6-10=11)$.

Para el segundo trimestre las unidades a producir son de 15 TM. Para este cálculo se ha tenido en cuenta que el inventario inicial del segundo trimestre es igual al inventario final del primer trimestre y llega a $6 \mathrm{TM}$. Como los requerimientos de ventas son $15 \mathrm{TM}$, 


\section{Pensamiento Crítico Vol. 20. Nº}

y el inventario final el $40 \%$ de los requerimientos del tercer trimestre $(0.40 \times 15=6)$, el cálculo sería: requerimientos de ventas más inventario final menos inventario inicial igual a 15 TM $(6+15-6=15)$.

Para el tercer trimestre las unidades a producir son de 15 TM. Para este cálculo se ha tenido en cuenta que el inventario inicial del tercer trimestre es igual al inventario final del segundo trimestre y llegan a $6 \mathrm{TM}$. Como los requerimientos de ventas son 15 $\mathrm{TM}$, y el inventario final el $40 \%$ de los requerimientos del cuarto trimestre $(0.40 \times 15$ $=6)$, el cálculo sería: requerimientos de ventas más inventario final menos inventario inicial igual a 15 TM $(6+15-6=15)$.

Para el cuarto trimestre las unidades a producir son de 19 TM. Para este cálculo se ha tenido en cuenta que el inventario inicial del cuarto trimestre es igual al inventario final del tercer trimestre y llega a $6 \mathrm{TM}$. Como los requerimientos de ventas son $15 \mathrm{TM}$, y el inventario final del cuarto trimestre debe ser igual al inventario inicial del año por política de la empresa que es $10 \mathrm{TM}$, el cálculo sería: Inventario final más requerimientos de ventas menos inventario inicial igual a 19 TM $(10+15-6=19)$.

Otro elemento importante para elaborar el presupuesto de producción es tener bien definidos los coeficientes técnicos de producción. En este caso se aprecia que para la mano de obra directa la relación técnica de producción es de 1 a 1 . Es decir para cada unidad producida necesitamos una hora hombre. Para los materiales directos la relación técnica de producción es 2 a1. Es decir por cada unidad producida necesitamos 2 unidades de materiales directos. Para los gastos indirectos de producción se está considerando un monto total para cada trimestre.

Como en general el costo de producción es igual a la mano de obra directa (MOD), más los materiales directos (MATD) más los gastos indirectos de fabricación (GIF) para cada trimestre tenemos:

\section{Primer Trimestre}

Unidades a producir $=11 \mathrm{TM}$

Mano de obra directa $=11 \mathrm{HH}$. Se considera la relación técnica 1 a 1 .

Materiales directos $=22$ TM. Se considera la relación técnica 2 a 1 . 


\section{Raimundo Pacheco Mexzon}

Gastos indirectos de fabricación o producción $=\$ 5$ por cada trimestre.

Costo unitario de mano de obra directa (hora hombre) $=1$

Costo unitario de materiales directos (Tonelada métrica) $=1.5$

\begin{tabular}{|l|c|ccc|}
\hline \multicolumn{1}{|c|}{ RUBROS } & UND & \multicolumn{3}{|c|}{ 1ER TRM } \\
& & Cu & Q & C \\
\hline Mano de Obra Directa (MOD) & HH & 1 & 11 & 11 \\
Materiales Directos (MATD) & TM & 1.5 & 22 & 33 \\
Gastos Indirectos de Fabricación (GIF) & $\$$ & & & 5 \\
\hline Costo de Producción. & & $\mathbf{4 . 4 5}$ & & $\mathbf{4 9}$ \\
\hline Inventario Inicial & TM & & 10 & \\
Requerimiento de Ventas & TM & & 15 & \\
Inventario Final & TM & & 6 & \\
\hline Unidades a producir & TM & & \\
\hline
\end{tabular}

Se observa que para el primer trimestre la empresa producirá 11 TM con un costo total de producción de 49 . El costo unitario ponderado de producción será 4.45 por TM $(49 \div 11=4.45)$

\section{Segundo Trimestre}

Unidades a producir $=15 \mathrm{TM}$

Mano de obra directa $=15 \mathrm{HH}$. Se considera la relación técnica 1 a 1 .

Materiales directos $=30 \mathrm{TM}$. Se considera la relación técnica 2 a 1 .

Gastos indirectos de fabricación o producción $=\$ 5$ por cada trimestre.

Costo unitario de mano de obra directa (hora hombre) $=1$

Costo unitario de materiales directos (Tonelada métrica) $=1.5$ 


\section{Pensamiento Crítico Vol. 20. NoI}

\begin{tabular}{|c|c|c|c|c|}
\hline \multirow[t]{2}{*}{ RUBROS } & \multirow[t]{2}{*}{ UND } & \multicolumn{3}{|c|}{ 2DO TRM } \\
\hline & & $\mathrm{Cu}$ & $\mathbf{Q}$ & $\mathbf{C}$ \\
\hline Mano de Obra Directa (MOD) & $\mathrm{HH}$ & 1 & 15 & 15 \\
\hline Materiales Directos (MATD) & TM & 1.5 & 30 & 45 \\
\hline Gastos Indirectos de Fabricación (GIF) & $\$$ & & & 5 \\
\hline Costo de Producción. & & 4.33 & & 65 \\
\hline Inventario Inicial & TM & & 6 & \\
\hline Requerimiento de Ventas & TM & & 15 & \\
\hline Inventario Final & TM & & 6 & \\
\hline Unidades a producir & TM & & 15 & \\
\hline
\end{tabular}

Se observa que para el segundo trimestre la empresa producirá 15 TM con un costo total de producción de 65. El costo unitario ponderado de producción será 4.33 por TM $(65 \div 15=4.33)$.

\section{Tercer Trimestre}

Unidades a producir $=15 \mathrm{TM}$

Mano de obra directa $=15 \mathrm{HH}$. Se considera la relación técnica 1 a 1 .

Materiales directos $=30 \mathrm{TM}$. Se considera la relación técnica 2 a 1 .

Gastos indirectos de fabricación o producción $=\$ 5$ por cada trimestre.

Costo unitario de mano de obra directa (hora hombre) $=1.5$

Costo unitario de materiales directos (Tonelada métrica) $=2$ 


\section{Raimundo Pacheco Mexzon}

\begin{tabular}{|c|c|c|c|c|}
\hline \multirow[t]{2}{*}{ RUBROS } & \multirow[t]{2}{*}{ UND } & \multicolumn{3}{|c|}{ 3ER TRM } \\
\hline & & $\mathrm{Cu}$ & $\mathbf{Q}$ & C \\
\hline Mano de Obra Directa (MOD) & $\mathrm{HH}$ & 1.5 & 15 & 22.5 \\
\hline Materiales Directos (MATD) & $\mathrm{TM}$ & 2 & 30 & 60 \\
\hline Gastos Indirectos de Fabricación (GIF) & $\$$ & & & 5 \\
\hline Costo de Producción. & & 5.83 & & 87.5 \\
\hline Inventario Inicial & $\mathrm{TM}$ & & 6 & \\
\hline Requerimiento de Ventas & TM & & 15 & \\
\hline Inventario Final & TM & & 6 & \\
\hline Unidades a producir & TM & & 15 & \\
\hline
\end{tabular}

Se observa que para el tercer trimestre la empresa producirá 15 TM con un costo total de producción de 87.5 . El costo unitario ponderado de producción será 5.83 por TM $(87.5 \div 15=5.83)$.

\section{Cuarto Trimestre}

Unidades a producir $=19 \mathrm{TM}$

Mano de obra directa $=19 \mathrm{HH}$. Se considera la relación técnica 1 a 1 .

Materiales directos $=38 \mathrm{TM}$. Se considera la relación técnica 2 a 1 .

Gastos indirectos de fabricación o producción $=\$ 5$ por cada trimestre.

Costo unitario de mano de obra directa (hora hombre) $=1.5$

Costo unitario de materiales directos (Tonelada métrica) $=2$ 


\section{Pensamiento Crítico Vol. 20. NoI}

\begin{tabular}{|c|c|c|c|c|}
\hline \multirow[t]{2}{*}{ RUBROS } & \multirow[t]{2}{*}{ UND } & \multicolumn{3}{|c|}{ 4TO TRM } \\
\hline & & $\mathrm{Cu}$ & $\mathbf{Q}$ & C \\
\hline Mano de Obra Directa (MOD) & $\mathrm{HH}$ & 1.5 & 19 & 28.5 \\
\hline Materiales Directos (MATD) & TM & 2 & 38 & 76 \\
\hline Gastos Indirectos de Fabricación (GIF) & $\$$ & & & 5 \\
\hline Costo de Producción. & & 5.76 & & 109.5 \\
\hline Inventario Inicial & TM & & 6 & \\
\hline Requerimiento de Ventas & TM & & 15 & \\
\hline Inventario Final & $\mathrm{TM}$ & & 10 & \\
\hline Unidades a producir & TM & & 19 & \\
\hline
\end{tabular}

Se observa que para el cuarto trimestre la empresa producirá 19 TM con un costo total de producción de 109.5. El costo unitario ponderado de producción será 5.76 por TM $(109.5 \div 19=5.76)$.

\section{Total del Año}

Unidades a producir $=60 \mathrm{TM}$

Mano de obra directa $=60 \mathrm{HH}$. Se considera la relación técnica 1 a 1 .

Materiales directos $=120$ TM. Se considera la relación técnica 2 a 1 .

Gastos indirectos de fabricación o producción $=\$ 20$ (\$5 por trimestre).

Costo unitario ponderado de mano de obra directa (hora hombre) $=1.28$

Costo unitario ponderado de materiales directos (Tonelada métrica) $=1.78$ 


\section{Raimundo Pacheco Mexzon}

\begin{tabular}{|c|c|c|c|c|}
\hline \multirow[t]{2}{*}{ RUBROS } & \multirow[t]{2}{*}{ UND } & \multicolumn{3}{|c|}{ TOTAL AÑO } \\
\hline & & $\mathbf{C u}$ & $\mathbf{Q}$ & C \\
\hline Mano de Obra Directa (MOD) & $\mathrm{HH}$ & 1.28 & 60 & 77 \\
\hline Materiales Directos (MATD) & $\mathrm{TM}$ & 1.78 & 120 & 214 \\
\hline Gastos Indirectos de Fabricación (GIF) & $\$$ & & & 20 \\
\hline Costo de Producción. & & 5.18 & 60 & 311 \\
\hline Inventario Inicial & $\mathrm{TM}$ & & 10 & \\
\hline Requerimiento de Ventas & TM & & 60 & \\
\hline Inventario Final & $\mathrm{TM}$ & & 10 & \\
\hline Unidades a producir & TM & & 60 & \\
\hline
\end{tabular}

Se observa que para el año la empresa producirá 60 TM con un costo total de producción de 311 . El costo unitario ponderado de producción para el año será 5.18 por TM $(311 \div 60=5.18)$.

Aparte del presupuesto de producción de bienes o servicios la empresa tiene gastos generales en cada una de las áreas que conforman su organización. Estos egresos o gastos se refieren a: gastos de personal, servicios de terceros, cargas diversas de gestión, cargas financieras entre otros. El presupuesto de gastos generales se puede resumir en el siguiente formato: 
PRESUPUESTO DE GASTOS GENERALES DEL AÑO

\begin{tabular}{|l|c|c|c|c|c|}
\hline \multicolumn{1}{|c|}{ DESCRIPCIÓN } & $\begin{array}{c}\text { 1ER } \\
\text { TRM }\end{array}$ & $\begin{array}{c}\text { 2DO } \\
\text { TRM }\end{array}$ & $\begin{array}{c}\text { 3ER } \\
\text { TRM }\end{array}$ & $\begin{array}{c}\text { 4TO } \\
\text { TRM }\end{array}$ & $\begin{array}{c}\text { TOTAL } \\
\text { AÑO }\end{array}$ \\
\hline Gastos de personal & 1 & 1 & 1 & 1 & 4 \\
\hline Sueldos y salarios & 0.5 & 0.5 & 0.5 & 0.5 & 2 \\
\hline Comisiones & 0.1 & 0.1 & 0.1 & 0.1 & 0.4 \\
\hline Gratificaciones & 0.2 & 0.2 & 0.2 & 0.2 & 0.8 \\
\hline Horas extras & 0.1 & 0.1 & 0.1 & 0.1 & 0.4 \\
\hline Viáticos & 0.05 & 0.05 & 0.05 & 0.05 & 0.2 \\
\hline Beneficios sociales & 0.05 & 0.05 & 0.05 & 0.05 & 0.2 \\
\hline Servicios terceros & 2 & 2 & 2 & 2 & 8 \\
\hline Fletes & 1 & 1 & 1 & 1 & 4 \\
\hline Combustibles & 0.4 & 0.4 & 0.4 & 0.4 & 1.6 \\
\hline Energía & 0.5 & 0.5 & 0.5 & 0.5 & 2 \\
\hline Seguros & 0.1 & 0.1 & 0.1 & 0.1 & 0.4 \\
\hline Tributos & & & & 3 & 3 \\
\hline Cargas diversas & 1.5 & 1.5 & 1.5 & 1.5 & 6 \\
\hline Alquileres & 0.6 & 0.6 & 0.6 & 0.6 & 2.4 \\
\hline Utiles de escritorio & 0.4 & 0.4 & 0.4 & 0.4 & 1.6 \\
\hline Teléfono & 0.3 & 0.3 & 0.3 & 0.3 & 1.2 \\
\hline Almacenaje & 0.1 & 0.1 & 0.1 & 0.1 & 0.4 \\
\hline Mantenimiento & 0.1 & 0.1 & 0.1 & 0.1 & 0.4 \\
\hline Cargas financieras & & 3.5 & & 3.5 & 7 \\
\hline Provisiones & & & & 1 & 1 \\
\hline Depreciación & & & & 0.8 & 0.8 \\
\hline Tiempo de servicios & & & & 0.2 & 0.2 \\
\hline TOTAL & $\mathbf{4 . 5}$ & $\mathbf{8}$ & $\mathbf{4 . 5}$ & $\mathbf{1 2}$ & $\mathbf{2 9}$ \\
\hline
\end{tabular}

Este total de gastos generales que llegan a 29 en el año están distribuidos en 7 de gastos financieros, 10 como gastos de venta y 12 como gastos administrativos. En resumen tenemos:

\begin{tabular}{|l|c|c|c|c|c|}
\hline \multicolumn{1}{|c|}{ RUBROS } & $\begin{array}{c}\text { 1ER } \\
\text { TRM }\end{array}$ & $\begin{array}{c}\text { 2DO } \\
\text { TRM }\end{array}$ & $\begin{array}{c}\text { 3ER } \\
\text { TRM }\end{array}$ & $\begin{array}{c}\text { 4TO } \\
\text { TRM }\end{array}$ & $\begin{array}{c}\text { TOTAL } \\
\text { AÑO }\end{array}$ \\
\hline Gastos de venta & 2.5 & 2.5 & 2.5 & 2.5 & 10 \\
\hline Gastos administrativos & 2 & 2 & 2 & 6 & 12 \\
\hline Gastos financieros & 0 & 3.5 & 0 & 3.5 & 7 \\
\hline TOTAL & $\mathbf{4 . 5}$ & $\mathbf{8}$ & $\mathbf{4 . 5}$ & $\mathbf{1 2}$ & $\mathbf{2 9}$ \\
\hline
\end{tabular}




\section{Raimundo Pacheco Mexzon}

Para calcular el presupuesto de ganancias y pérdidas debemos de tener en cuenta el gráfico 1.

Como se puede apreciar los ingresos por ventas los tenemos calculados en el presupuesto de ventas y para el año llegan a 350.

El costo de producción está calculado en el presupuesto de producción y llega a 311.

Los gastos operativos que están constituidos por los gastos de venta y los gastos administrativos se encuentran calculados en el presupuesto de gastos generales. Para el gasto de ventas tenemos 10 y para los gastos administrativos 12 .

Los gastos financieros también aparecen en el presupuesto de gastos generales y llegan a 7.

Gráfico 1

\section{ESTRUCTURACIÓN DEL PRESUPUESTO DE GANANCIAS Y PÉRDIDAS}

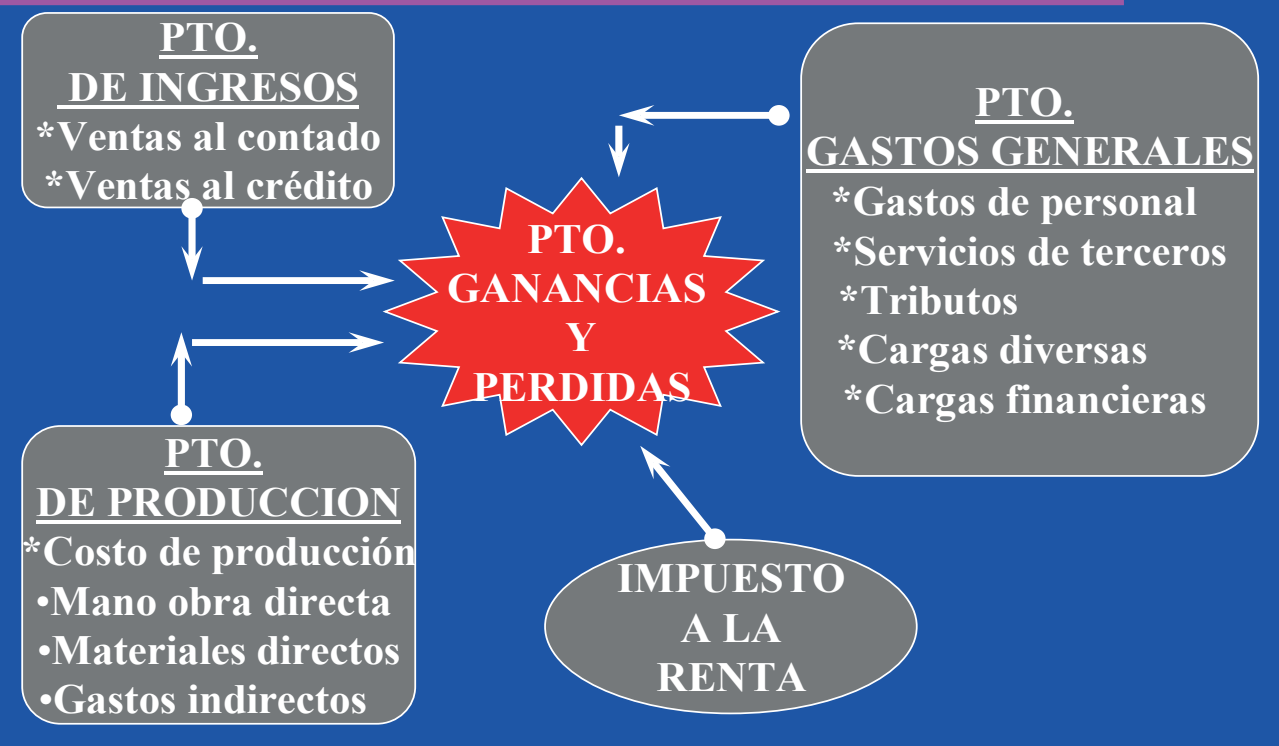




\section{Pensamiento Crítico Vol. 20. No I}

Con esta información podemos estructurar el presupuesto de ganancias y pérdidas tal conforme se indica a continuación:

\section{PRESUPUESTO DE GANANCIAS Y PÉRDIDAS}

\begin{tabular}{|c|c|}
\hline \multicolumn{1}{|c|}{ Ingreso por Ventas } & $\mathbf{3 5 0}$ \\
\hline Menos Costo de ventas & 311 \\
\hline Utilidad Bruta & $\mathbf{3 9}$ \\
\hline Menos Gastos Operativos & 22 \\
\hline Gastos de venta & 10 \\
\hline Gastos administrativos & 12 \\
\hline Utilidad de Operaciones & $\mathbf{1 7}$ \\
\hline Menos Gastos financieros & 7 \\
\hline Utilidad antes de impuestos & $\mathbf{1 0}$ \\
\hline Impuesto a la renta (28\%) & 2.8 \\
\hline Utilidad neta & $\mathbf{7 . 2}$ \\
\hline
\end{tabular}

NOTA: Como el costo de ventas es igual al costo de producción más el inventario inicial menos el inventario final de productos terminados se está dando el caso que el costo de ventas es igual al costo de producción porque por política de la empresa el inventario inicial y el inventario final en el año es el mismo. Todo lo que se produce se vende.

Veamos con los datos que tenemos:

- Inventario inicial 10

- Inventario final 10

- Producción 60

- Costo de Producción 311 


\section{Raimundo Pacheco Mexzon}

Costo de ventas $=$ Costo de producción + Inventario inicial - Inventario final

Costo de ventas $=311+10-10$

\section{Costo de ventas $=311$}

\section{Cash Flow o Flujo de Caja}

Para elaborar el Flujo de Caja o Cash Flow tomamos en cuenta la información que nos proporciona el Plan de Utilidades o presupuesto operativo de la empresa, las inversiones que están definidas en el presupuesto de capital y los otros rubros como la parte corriente de la deuda de mediano y largo plazo que se vencen en el periodo actual.

El siguiente gráfico nos muestra como se relacionan estos conceptos para elaborar el Cash Flow o Flujo de Caja.

Del presupuesto de capital o de inversiones de la empresa se ha definido invertir

14 en el año: 4 para el primer trimestre, 4 para el segundo, 3 para el tercero y 3 para el cuarto.

De la deuda de mediano y largo plazo se tiene que pagar en el segundo trimestre 2 y en el cuarto 3.

El saldo inicial de caja que aparece en el Balance General del año anterior es 3.5.

Por política de la empresa las ventas al crédito son a 90 días de tal manera que lo que se vende en el primer trimestre se cobra en el segundo. Lo que se vende en el segundo se cobra en el tercero. Lo que se vende en el tercero se cobra en el cuarto y lo que se vende en el cuarto se cobra en el primero del próximo año. Para este caso se estima que las ventas al crédito del cuarto trimestre del año anterior se cobran en el primer trimestre de este año y es igual a 30. 


\section{ESTRUCTURACION DEL PRESUPUESTO DE CAJA ( CASH FLOW)}

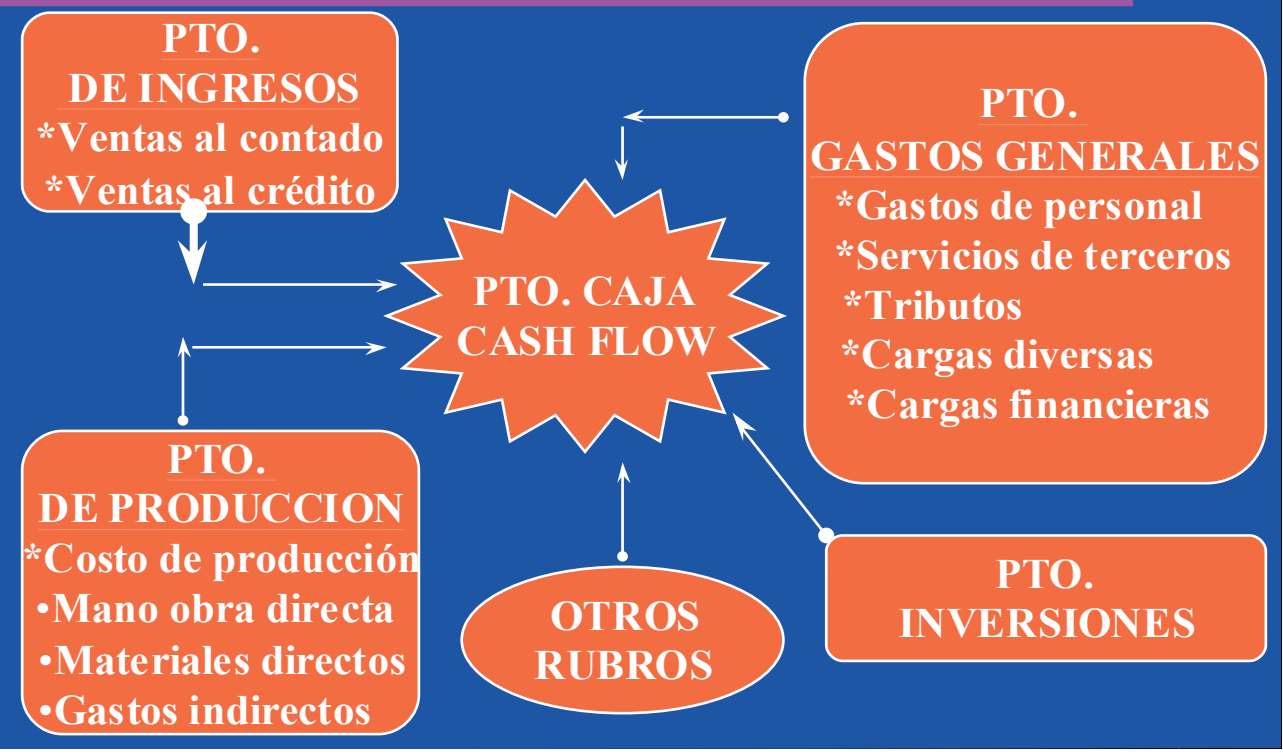

Con esta información el formato que se puede utilizar es el siguiente:

CASH FLOW O FLUJO DE CAJA

\begin{tabular}{|l|l|l|l|l|l|}
\hline \multicolumn{1}{|c|}{ RUBROS } & \multicolumn{1}{|c|}{$\begin{array}{c}\text { 1ER } \\
\text { TRM }\end{array}$} & \multicolumn{1}{|c|}{$\begin{array}{c}\text { 2DO } \\
\text { TRM }\end{array}$} & \multicolumn{1}{|c|}{$\begin{array}{c}\text { 3ER } \\
\text { TRM }\end{array}$} & \multicolumn{1}{|c|}{$\begin{array}{c}\text { 4TO } \\
\text { TRM }\end{array}$} & $\begin{array}{c}\text { TOTAL } \\
\text { ANO }\end{array}$ \\
\hline Ingresos Operativos & $\mathbf{8 0}$ & $\mathbf{8 0}$ & $\mathbf{9 5}$ & $\mathbf{9 5}$ & $\mathbf{3 5 0}$ \\
\hline Ventas al contado & 50 & 50 & 60 & 60 & 220 \\
\hline Ventas al crédito & 30 & 30 & 35 & 35 & 130 \\
\hline Egresos Operativos & $\mathbf{5 3 . 5}$ & $\mathbf{7 3}$ & $\mathbf{9 2}$ & $\mathbf{1 2 0 . 5}$ & $\mathbf{3 3 9}$ \\
\hline Mano de obra directa & 11 & 15 & 22.5 & 28.5 & 77 \\
\hline
\end{tabular}




\section{Raimundo Pacheco Mexzon}

\begin{tabular}{|c|c|c|c|c|c|}
\hline Materiales Directos & 33 & 45 & 60 & 76 & 214 \\
\hline Gasto indirectos de Fabri. & 5 & 5 & 5 & 5 & 20 \\
\hline Gastos de personal & 1 & 1 & 1 & 1 & 4 \\
\hline Servicios de terceros & 2 & 2 & 2 & 2 & 8 \\
\hline Tributos & & & & 3 & 3 \\
\hline Cargas diversas & 1.5 & 1.5 & 1.5 & 1.5 & 6 \\
\hline Cargas financieras & & 3.5 & & 3.5 & 7 \\
\hline Ingre - Egre Operativos & 26.5 & 7 & 3 & -25.5 & 11 \\
\hline Pago deuda MyL plazo & & 2 & & 3 & 5 \\
\hline Inversiones & 4 & 4 & 3 & 3 & 14 \\
\hline Ingre - Egre Totales & 22.5 & $\mathbf{1}$ & $\mathbf{0}$ & -31.5 & -8 \\
\hline Saldo Inicial & 3.5 & 26 & 27 & 27 & 3.5 \\
\hline Saldo Final & 26 & 27 & 27 & -4.5 & -4.5 \\
\hline
\end{tabular}

Observamos que operativamente la empresa está generando caja positiva en el año por un total de 11. Esto nos indica que las operaciones de la empresa se financian con recursos generados por la propia empresa.

Sin embargo como en el cuarto trimestre, al considerar las inversiones, el saldo final de caja es negativo en 4.5 (- 4.5), el Gerente financiero debe preocuparse de financiarlo con recursos de terceros vía préstamos o aportes de capital de los dueños.

\section{Conclusiones}

El presupuesto operativo o plan de utilidades es la base para elaborar el Cash Flow o Flujo de Caja Operativo de la empresa. 


\section{Pensamiento Crítico Vol. 20. NoI}

Considerando las inversiones que se indican en el presupuesto de capital así como el pago de las deudas procedentes del mediano y largo plazo se prepara el Cash Flow o Flujo de Caja General de la empresa.

El Cash Flow se constituye en una herramienta muy importante para la gestión financiera porque muestra en qué momento el gerente financiero necesita conseguir fondos para garantizar la ejecución de las operaciones e inversiones de la empresa. Así como pagar sus deudas oportunamente.

\section{Recomendaciones}

Preparar el presupuesto de caja (Cash Flow o Flujo de Caja) para cada año desagregándolo mensualmente para tener la información más desagregada y más reciente para la oportuna toma de decisiones.

Divulgar el concepto de que el Cash Flow es una herramienta muy importante en la toma de decisiones financieras y que su elaboración parte del Plan de Utilidades de la empresa.

\section{Referencias bibliográficas}

Córdova Padilla, Marcial. (2007). Gerencia Financiera Empresarial, ECOE Ediciones.

Ehrhardt Michael C. (2007). Eugene F. Brigham. Finanzas Corporativas. CENEGAGE Learning.

Hartley W.C.F. (2008). Cash Flow: Su Planificación y Control, Editorial DEUSTO.

J.A. FORSYTH. (2006). Finanzas Empresariales.

Pacheco Mexzon, Raymundo. (2014). Gerencia Financiera, IIE UNMSM.

Ross. (2005). Westerfield. Jaffe. Finanzas Corporativas. McGraw-Hill. 